\title{
On the Simulation of the Influence of Defects on Immersed Plane Periodic Multilayer Viscoelastic Media
}

\author{
Massaï Hatoumva ${ }^{1}$, Emmanuel Siryabe ${ }^{2 *}$, Pierre Marechal ${ }^{3}$, \\ Guy Edgar Ntamack ${ }^{4}$, Gambo Betchewe ${ }^{1}$
}

${ }^{1}$ Faculté des Sciences, Département de Physique, Université de Maroua, Maroua, Cameroon

${ }^{2}$ Safran Helicopter Engines, Groupe Evaluation Non Destructive, Bordes, France

${ }^{3}$ Laboratoire Ondes et Milieux Complexes (LOMC), UMR 6294 CNRS, Université du Havre, Le Havre, France

${ }^{4}$ Groupe de Mécanique, Matériaux et Acoustique, P.B: 454, Département de Physique, Faculté des Sciences, Université de

Ngaoundéré, Ngaoundéré, Cameroon

Email: *emmanuel.siryabe@safrangroup.com

How to cite this paper: Hatoumva, M. Siryabe, E., Marechal, P., Ntamack, G.E. and Betchewe, G. (2021) On the Simulation of the Influence of Defects on Immersed Plane Periodic Multilayer Viscoelastic Media. Open Journal of Acoustics, 11, 17-30. https://doi.org/10.4236/oja.2021.112002

Received: May 7, 2021

Accepted: June 15, 2021

Published: June 18, 2021

Copyright $\odot 2021$ by author(s) and Scientific Research Publishing Inc. This work is licensed under the Creative Commons Attribution International License (CC BY 4.0).

http://creativecommons.org/licenses/by/4.0/

\begin{abstract}
This work deals with the study of a plane periodic multilayer structure in which the elementary stack consists of two plates in contact: one in aluminum $(\mathrm{AL})$ and the other one in polyethylene (PE). These isotropic materials, present a high acoustic impedance contrast. The attenuation of the longitudinal and transverse waves is taken into account in the polyethylene but neglected in the aluminum plate. The effect of different defects is analyzed. Firstly, we focus on the effect of the presence of grease inclusion in the polyethylene plate (considering the two plates of the elementary stack in perfect contact). Secondly, the effect of disbond simulated by the insertion of a thin Teflon layer between the interfaces of the two layers constituting the elementary stack of the multilayer structure is investigated. Finally, the effect of the stacking sequences of the multilayer is analyzed. In order to obtain the effective acoustic parameters of polyethylene layer, allowing to evaluate the reflection and transmission coefficients using the stiffness matrix method developed by Rokhlin et al., four homogenization models are analyzed, then the best one to our configuration is chosen. The comparison of the simulation results is carried out.
\end{abstract}

\section{Keywords}

Reflection Coefficients, Transmission Coefficients, Defects, Multilayer, Periodic Structure 


\section{Introduction}

Periodic multi-layered media are widely studied because of their very interesting properties such as exhibiting absolute or local band gaps in their dispersion curves. The width and position of these bands depend on the physical and geometric characteristics of the materials and their frequency. Indeed, by the modification of the physical or geometric properties of a material constituting one or more periods, the width and the position of the forbidden or passbands can be modified, and very fine bandwidths can appear in the prohibited bands. It is thus possible to perform more or less selective wave filtering depending on the intended application [1] [2]. Numerous theoretical methods can be used to obtain the forbidden and passbands of the periodic media. Our theoretical approach is based on the stiffness matrix [3] method which ensures unconditional numerical stability, whatever the frequency range and the incidence angle. This method allows to study the forbidden and passbands from the calculation of the reflection and transmission coefficients. In the literature, several studies can be found concerning the study of periodic multilayer media [4] [5] [6]. For example, Lenoir et al. [4] [5], studied two cases of insonation and showed that, if we consider attenuation in one of the constitutive plates (polyethylene), the reflection coefficients are different depending on the insonation side while the transmission coefficients are identical. Maréchal et al. [7] showed that the number of periods necessary to obtain stable forbidden and passbands depends on the geometrical and physical characteristics of the viscoelastic plate. For their configurations studied, they demonstrated that three periods $(N=3)$ ensure the convergence of the reflection spectrum (or transmission spectrum). They have also shown that [8] the importance that should be given to the thickness measurement and the characterization of the viscoelastic properties of the materials used as input data for the multilayer structure. Siryabe et al. [6] compared results of multilayer without or with hole configurations and showed that throughout holes in the polyethylene plate allow the rapid observation of forbidden bands. Lenoir et al. [9] studied the influence of defect layers on the reflection coefficients on a plane multilayered fluid-loaded structure composed of $\mathrm{N}$ periods. Two types of inserted flaws are considered, either inside a period by varying the polyethylene layer thickness (without taking into account the attenuation), or by inserting a defect layer. The former case is based on experimental ascertainments which imply to take into account thickness variations. In the latter case, a layer whose acoustic impedance is different from those of the $\mathrm{Al}$ and PE plates plays the role of the defect. The defect layer is either in copper or in glass. Particular attention has been paid to the reflection minimums at low frequency, these ones being linked to the so-called vertical modes. They showed the linearity of the sensitivity of the vertical modes to thickness variations. In the case of the insertion of a defect layer, a very narrow passband emerge in a wide stopband, at a frequency depending on the thickness and acoustical properties of the defect layer. More recently, Khaled et al. [10] investigated the influence of the insertion 
of two geometric defect layers in a 1D periodic structure composed of $N$ elementary cells, through the analysis of the band gaps and passbands of the transmission coefficient. The concept behind the introduction of a defect into the multilayer stacks is a way to create the so-called defect modes or resonant modes. The numerical study is carried out in the case of $N$-periods made of a Glass isotropic layer and another one of fluid water. The variations of the resonant modes in terms of the defect locations and thicknesses are shown as graphs.

The present study is the contribution to the previous work on multilayer periodic structure in which defects were introduced [9] [10]. We consider plane periodic multilayers composed of $N$ periods $(1 \leq N \leq 3)$. The elementary stack is made of two plates, one in aluminum and the other one in polyethylene, both having the same thickness, and presenting a high acoustic impedance contrast. Simulations are made at normal incidence. Contrary to the previous studies [6] [9] [10] concerning periodic structures, we will consider firstly, the presence of inclusion matter in the polyethylene plate, secondly the effect of boundary conditions different from perfect ones between the two plates composing a period and finally the presence of more than two layers in a stack. Theoretical basis used to obtain effective acoustic properties and reflection, transmission and absorption coefficients are described. Then, the results for the case of polyethylene containing grease inclusions configurations are compared with those of air porosities [6] and without porosities [4]. The effect of disbond simulated by the insertion of a thin Teflon layer and the number of layers composing a period are discussed. Finally, conclusion and some prospects are presented.

\section{Theoretical Basis}

\subsection{Context}

The geometry of the problem (Figure 1(a)) consists with a stacking of $N$ periods $(1 \leq N \leq 3)$, each one made up of two isotropic plates of different acoustic properties. As a case of study (Figure 1(b)), Siryabe et al. investigated the AL/PEp stack, where the polyethylene plate (PE) was perforated (air porosities) [6]. In this study, as an extension, we consider the case of grease inclusions. The aim is to observe the effect of the presence of grease on the reflection and transmission coefficients of the considered multilayer. Here, one will note (PEg) for polyethylene with grease. In both cases, the longitudinal and transverse waves attenuations are taken into account in the polyethylene plate but neglected in the aluminum one [4].

\subsection{Homogenization Methods}

Since the polyethylene layer was considered as containing grease inclusions after manufacturing, thus, it is suitable to determine its new effective properties using homogenization models as was done in [6]. In order to retain the appropriate model to our study, a comparison of the approaches suggested by Voigt [11] [12], Gaunaurd (G-sph) [13] and Hashin-Shtrikman (HS-iso, HS-ort) [14] [15] 


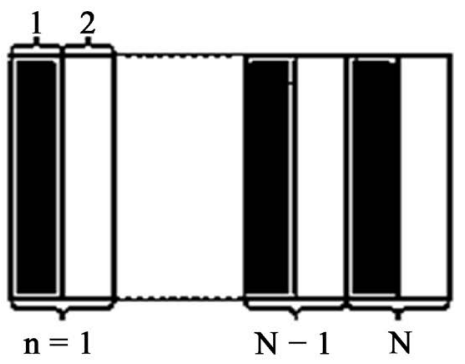

(a)

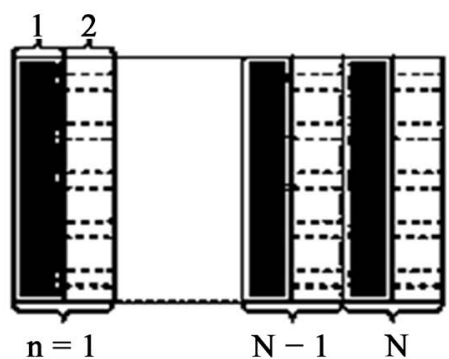

(b)

(c)

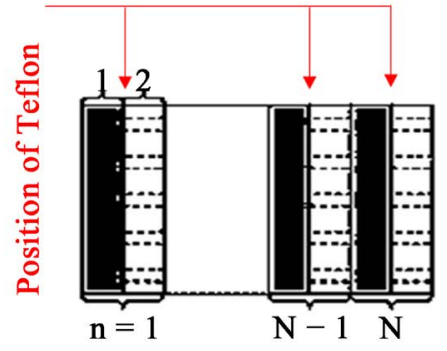

Figure 1. (a) Geometry of the problem, (b) PE with spherical or cylindrical grease inclusions and (c) position of the insertion of thin Teflon plate.

is made. For a plate with inclusions, the estimation of effective properties can be done using the following relations [6]:

$$
\left\{\begin{array}{l}
E_{\text {eff }}=\frac{9 K_{\text {eff }} G_{\text {eff }}}{3 K_{\text {eff }}+G_{\text {eff }}} \\
v_{\text {eff }}=\frac{3 K_{\text {eff }}-2 G_{\text {eff }}}{6 K_{\text {eff }}+2 G_{\text {eff }}}
\end{array}\right.
$$

were $\left\{K_{\text {eff }} G_{\text {eff }}\right\}$ are given by homogenization models in Table 1.

The longitudinal and transverse effective velocities $\left\{c_{L, e f f} c_{T, e f f}\right\}$ which constitute the effective acoustic properties of a plate with inclusion, the effective density $\rho_{\text {eff }}$ and the effective acoustic impedance $Z_{L, \text { eff }}$ are respectively expressed by [6]:

$$
\left\{\begin{array}{l}
c_{\text {Leff }}=\sqrt{\frac{E_{\text {eff }} \cdot\left(1-v_{\text {eff }}\right)}{\rho_{\text {eff }} \cdot\left(1+v_{\text {eff }}\right)\left(1-2 v_{\text {eff }}\right)}} \\
c_{\text {Teff }}=\sqrt{\frac{E_{\text {eff }}}{2 \rho_{\text {eff }} \cdot\left(1+v_{\text {eff }}\right)}}
\end{array}\right.
$$

and

$$
\left\{\begin{array}{l}
\rho_{\text {eff }}=\rho+V_{f}\left(\rho_{i}-\rho\right) \\
Z_{L, \text { eff }}=\rho_{\text {eff }} C_{L, \text { eff }}
\end{array}\right.
$$

In a general way, depending on the homogenization model, the effective mechanical properties $X_{\text {eff }}$ of a heterogeneous material can be written as follows:

$$
X_{\text {eff }}=\frac{X+a+b V_{f}\left(X_{i}-X\right)}{1+m+x V_{f}}
$$


Table 1. Effective mechanical properties according to the homogenization models.

\begin{tabular}{|c|c|c|}
\hline Model & $K_{\text {eff }}$ & $G_{\text {eff }}$ \\
\hline Voigt & $K+\left(K_{g}-K\right) V_{f}$ & $G+\left(G_{g}-G\right) V_{f}$ \\
\hline \multirow[b]{2}{*}{ G-sph } & $K+\frac{4 G}{3 K_{g}+4 G}\left(K_{g}-K\right) V_{f}$ & $G+\frac{14}{3} G_{g}+\left(G_{g}-G\right) V_{f}$ \\
\hline & $1-\frac{3 K_{g}-3 K}{3 K_{g}+4 G} V_{f}$ & $\overline{1-\frac{7}{8} \frac{G_{g}}{G}+\frac{7}{8}\left(1-\frac{G_{g}}{G}\right) V_{f}}$ \\
\hline \multirow[b]{2}{*}{ HS-iso } & $K+\frac{4 G}{3 K_{g}+4 G}\left(K_{g}-K\right) V_{f}$ & $G+\frac{6 G_{g}(K+2 G)}{9 K+8 G}+\left(G_{g}-G\right) V_{f}$ \\
\hline & $1-\frac{3 K_{g}-3 K}{3 K_{g}+4 G} V_{f}$ & $1+\frac{6 G_{g}(K+2 G)}{G(9 K+8 G)}+\frac{6(K+2 G)}{9 K+8 G}\left(1-\frac{G_{g}}{G}\right) V_{f}$ \\
\hline HS-ort & $K+\frac{G}{K_{g}+G}\left(K_{g}-K\right) V_{f}$ & $G+\frac{K G\left(G_{g}-G\right)}{K\left(G_{g}+G\right)+2 G G_{g}} V_{f}$ \\
\hline & $1-\frac{K_{g}-K}{K_{g}+G} V_{f}$ & $1-\frac{(K+2 G)\left(G_{g}-G\right)}{K\left(G_{g}+G\right)+2 G G_{g}} V_{f}$ \\
\hline
\end{tabular}

$\rho$ : Polyethylene's density; $K$ : Polyethylene bulk modulus; $G$ : Polyethylene shear modulus; $\rho_{g}$ Grease's density; $K_{g}$ : Grease bulk modulus; $G_{g}$ : Grease shear modulus; $V_{\dot{f}}$ Volume fraction.

where $\{x, a, b, m\}$ are either a constant or function depending on the homogenization model, associated to the elastic properties of the matrix $X=\{K, G, \rho\}$ and $V_{f}$ is the volume fraction of inclusions having the mechanical properties $X_{i}=\left\{K_{D}\right.$ $G_{i p} \rho_{i}$.

Since the effective density $\rho_{\text {eff }}$ is considered as that given in Equation (3), the effective properties $\left\{K_{e f f} G_{e f f}\right\}$ are summarized in Table 1 according to the different homogenization models for a polyethylene plate containing grease inclusions.

\subsection{Comparison of Homogenization Models}

In this part, the equivalent modulus curves according to the volume fraction $V_{f}$ are plotted for the four models. The normalized $\left\{K_{\text {eff }} G_{\text {eff }}\right\}$ coefficients, i.e. $\left\{K_{\text {eff }} / K\right.$, $G_{e f f} G$ are shown to decrease when $V_{f}$ increases in Figure 2(a) and Figure 2(b), respectively.

This reduction depends on the homogenization model. Moreover, we observed that the Hashin-Shtrikman model for orthotropic inclusions (HS-ort) and isotropic inclusions (HS-iso) gives values of effective mechanical properties lower than those obtained with other models. Consequently, the effective acoustic properties $\left\{c_{L, \text { eff }} c_{T, e f f}\right\}$ required also will be lowest possible: what will make it possible as well as possible to describe the behavior of the wave in the structure with porosities (see Figure 3(a) and Figure 3(b), respectively).

For the polyethylene plate containing grease inclusions, the new values of the effective properties for longitudinal and transverse velocities (Equation (2)), density and acoustic impedance (Equation (3) for the four models are calculated. The results are presented in Table 2.

As we can see, the effective acoustic properties for the HS-ort model are very 


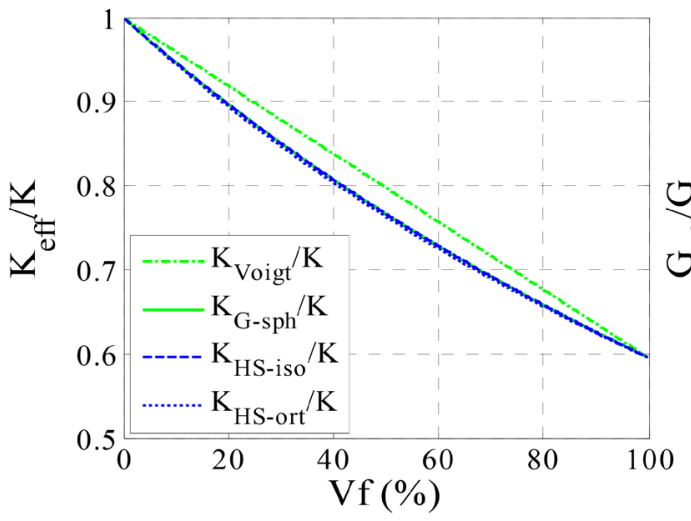

(a)

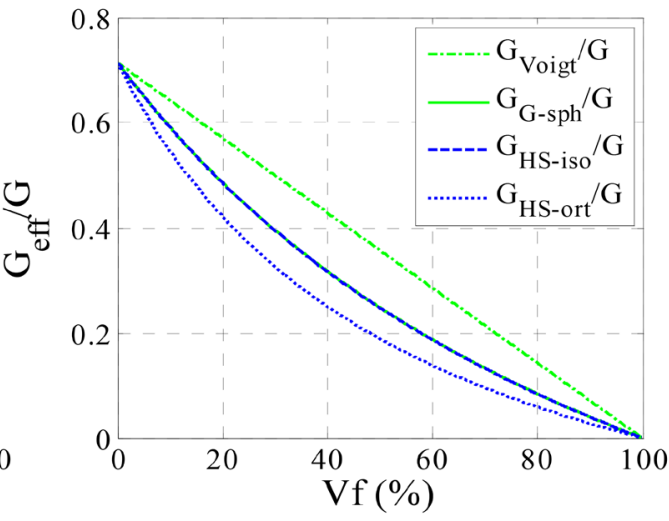

(b)

Figure 2. Normalized elastic properties deduced from (Voigt, G-sph, HS-Iso, HS-ort) homogenization models according to the volume fraction $V_{\dot{f}}$ (a) normalized effective bulk modulus $K_{\text {eff }} / K$ and (b) normalized effective shear modulus $G_{e f f} /$.

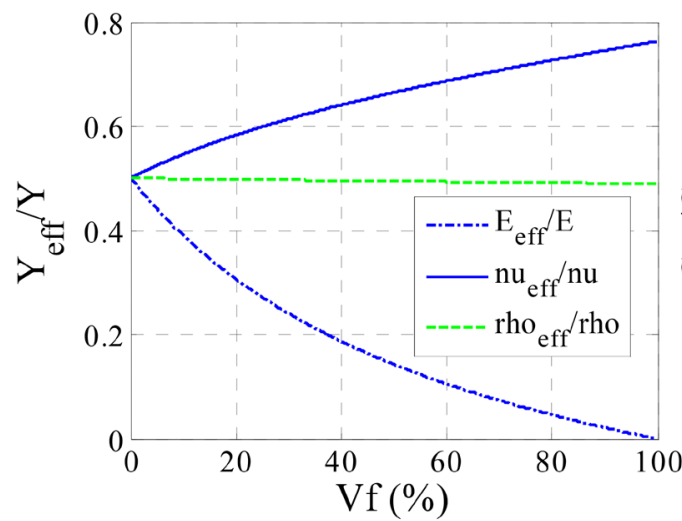

(a)

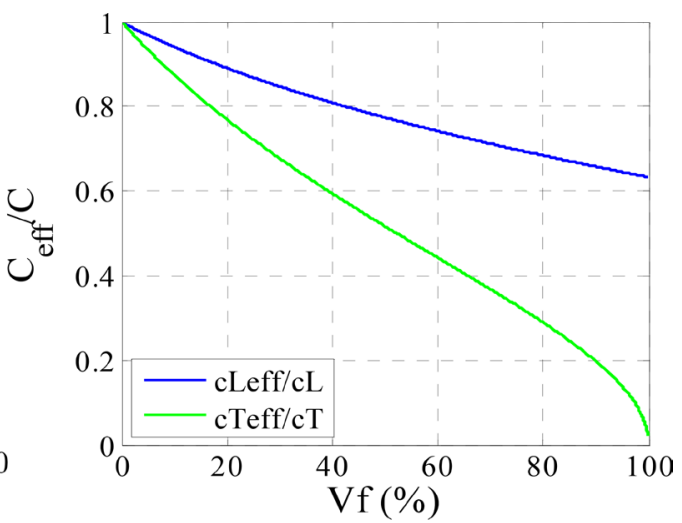

(b)

Figure 3. Effective elastic and acoustic properties deduced from HS-ort homogenization model as a function of the volume fraction $V_{\dot{F}}$ (a) normalized Young modulus, Poisson's ratio and density $\left\{E_{\text {eff }}\right.$ $\left.v_{\text {eff }} \rho_{\text {eff }}\right\}$ and (b) normalized longitudinal and transverse velocities $\left\{c_{L, \text { eff }} c_{T, \text { eff }}\right\}$.

Table 2. Effective acoustic properties according to the homogenization models for the polyethylene plate with $20 \%$ grease inclusion (PEg).

\begin{tabular}{ccccc}
\hline Model & $\rho_{\text {eff }}\left(\mathrm{kg} / \mathrm{m}^{3}\right)$ & $c_{L, \text { eff }}(\mathrm{m} / \mathrm{s})$ & $c_{T, \text { eff }}(\mathrm{m} / \mathrm{s})$ & $Z_{L, \text { eff }}(\mathrm{MRa})$ \\
\hline Voigt & 936 & 2215 & 1074 & 2.07 \\
G-sph & 936 & 2176 & 991 & 2.04 \\
HS-iso & 936 & 2176 & 991 & 2.04 \\
HS-ort & 936 & 2133 & 925 & 1.99 \\
\hline
\end{tabular}

$\rho_{\text {eff }}\left(\mathrm{kg} / \mathrm{m}^{3}\right):$ Density; $\left\{\begin{array}{lll}c_{L, \text { eff }} & c_{T, e f f}\end{array}\right\}(\mathrm{m} / \mathrm{s})$ : Longitudinal and transverse wave velocities; $Z_{L, \text { eff }}(\mathrm{MRa}):$ Longitudinal acoustic impedance.

lower than effective acoustic properties for the HS-iso model. Thus, in the following, since orthotropic inclusions are considered, effective acoustic properties calculated using Hashin-Shtrikman (HS-ort) model will be retained and will be useful as input data for simulations. 


\subsection{Stiffness Matrix Method (SMM)}

We consider two configurations of insonation: direct insonation (made from the aluminum layer side) and reverse insonation (from polyethylene side). For the direct case, the obtained reflection coefficient is noted $R_{d}$ and for the reverse one, the reflection coefficient is noted $R_{r}$. When attenuation is considered in the multilayer structure, those reflection coefficients are different according to the insonation side, either in the direct case $R_{d}$ or in the reverse case $R_{r}$, whereas the transmission coefficient $T$ is identical whatever the insonation side. These coefficients are obtained from a procedure suggested by Rokhlin et al. [3] [16]. For each period $N$, one defines a $(4 \times 4)$ flexibility matrix:

$$
S_{n}=\left[\begin{array}{ll}
S_{11} & S_{12} \\
S_{21} & S_{22}
\end{array}\right]_{n}
$$

where $S_{i j}$ are $2 \times 2$ submatrices.

The $S_{i j}$ submatrices for $N$ periods are deduced from the ones for $(N-1)$ periods and those of an additional $n$ layer by the following recursive relationships:

$$
\left\{\begin{array}{l}
S_{11}^{N}=S_{11}^{N-1}+S_{12}^{N-1}\left(S_{11}^{n}-S_{22}^{N-1}\right)^{-1} S_{21}^{N-1} \\
S_{12}^{N}=-S_{12}^{N-1}\left(S_{11}^{n}-S_{22}^{N-1}\right)^{-1} S_{12}^{n} \\
S_{21}^{N}=S_{21}^{n}\left(S_{11}^{n}-S_{22}^{N-1}\right)^{-1} S_{21}^{N-1} \\
S_{22}^{N}=S_{22}^{n}-S_{21}^{n}\left(S_{11}^{n}-S_{22}^{N-1}\right)^{-1} S_{21}^{n}
\end{array}\right.
$$

It is shown that the expressions of $R_{d} R_{r}$ and $T$ coefficients are expressed according to the elements $(2,2)$ of the $S_{i j}^{N}$ submatrices extracted from global compliance matrix $S^{\mathrm{N}}$, denoted as $s_{i j}^{22}$ into the forms:

$$
\left\{\begin{array}{l}
R_{d}=\frac{\left(s_{11}^{22}-y_{F}\right)\left(s_{22}^{22}-y_{F}\right)+\left(s_{12}^{22}\right)^{2}}{\left(s_{11}^{22}+y_{F}\right)\left(s_{22}^{22}-y_{F}\right)+\left(s_{12}^{22}\right)^{2}} \\
R_{r}=\frac{\left(s_{11}^{22}+y_{F}\right)\left(s_{22}^{22}+y_{F}\right)+\left(s_{12}^{22}\right)^{2}}{\left(s_{11}^{22}+y_{F}\right)\left(s_{22}^{22}-y_{F}\right)+\left(s_{12}^{22}\right)^{2}} \\
T=\frac{2 y_{F} s_{12}^{22}}{\left(s_{11}^{22}+y_{F}\right)\left(s_{22}^{22}-y_{F}\right)+\left(s_{12}^{22}\right)^{2}}
\end{array}\right.
$$

where $y_{F}=\cos \theta /\left(j \omega Z_{F}\right)$.

\section{Results and Discussions}

In this section, the theoretical reflection, transmission and attenuation spectra are plotted according to the insonation side. The multilayer structure is immerged in a coupling medium (water in our case). In Table 3 are summarized the characteristics of each layer constituting the period and those of the coupling medium. Among acoustic properties, the effective properties of the air porosities polyethylene plate and those of the grease inclusions polyethylene plate are 
Table 3. Acoustic properties of each layer constituting a period and the propagation medium.

\begin{tabular}{ccccc}
\hline Medium & $\rho\left(\mathrm{kg} / \mathrm{m}^{3}\right)$ & $c_{L}(\mathrm{~m} / \mathrm{s})$ & $c_{T}(\mathrm{~m} / \mathrm{s})$ & $Z_{L}(\mathrm{MRa})$ \\
\hline AL & 2800 & 6380 & 3100 & 17.9 \\
PE & 940 & 2370 & 1200 & 2.23 \\
Teflon & 2200 & 1350 & - & 2.97 \\
Water & 1000 & 1480 & - & 1.48 \\
Grease & 920 & 1450 & - & 1.33 \\
\hline
\end{tabular}

$\rho\left(\mathrm{kg} / \mathrm{m}^{3}\right)$ : Density; $\left\{c_{L}, c_{T}\right\}(\mathrm{m} / \mathrm{s})$ : Longitudinal and transverse wave velocities; $Z_{L}(\mathrm{MRa})$ : Longitudinal acoustic impedance.

calculated using the HS-ort homogenization model presented in Table 2. The considered volume fraction was evaluated on the basis of grease inclusions, distributed in a random way into the thickness of the polyethylene plate. The diameter of each inclusion was considered negligible compared to the plate thickness. The Poisson's ratio of PE material is $v=1 / 3$ and the chosen volume fraction is $V_{f}=20 \%$. The chosen bandwidth frequency is ranging in $[0.75 ; 3.25]$ $\mathrm{MHz}$.

The constitutive layers of periods have each one $4 \mathrm{~mm}$ thickness and are denoted by AL, PE, PEp and PEg for aluminum, polyethylene, perforated polyethylene and polyethylene with grease inclusions, respectively. A computation carried out with plates without losses will give an identical reflection spectrum whatever the insonation side [4] [5]. For that, we take into account longitudinal absorption in the polyethylene, by considering that the attenuation is linearly dependent on the frequency: $\alpha=\alpha_{0} \cdot\left(f / f_{0}\right)$. This implies that the longitudinal wave velocity as a complex can be expressed as: $c_{L}=c_{L 0} \cdot\left(1-j \cdot \delta_{c L}\right)$, where the losses are estimated around $\delta_{c L}=\delta_{c L e f f}=1 \%$ in the polyethylene plate.

\subsection{Grease Inclusions Effect}

In previous works, Lenoir et al. [4] for ( $\mathrm{AL} / \mathrm{PE})$ periodic structures and Siryabe et al. [6] for (AL/PEp) periodic structures showed that reflection coefficients reach their final shape as from the second period $(N=2)$. It is also the case of our configuration (AL/PEg) (Figure 4(a) and Figure 4(b)). In addition, the reflection coefficients minima tend towards zero and the undulations in both $\mathrm{AL} / \mathrm{PEp}$ and $\mathrm{AL} / \mathrm{PEg}$ configurations disappear for $N=1$ period. A small shift and interchange is observed on local maxima and minima of reflection coefficients.

In the case of $\mathrm{PEg} / \mathrm{AL}$ configuration (Figure 4(b)), undulations related to the vibrations of the polyethylene layer are obtained at the period $N=1$. We also observed a very great decreasing in the amplitude of the reflection coefficients as the frequency increases. This result was also obtained by Siryabe et al. [6] in the case of PEp/AL. Concerning the minima of reflection; they tend towards zero for the three configurations whatever the frequency and the number of periods.

The difference of spectrum according to the insonation side is also observed 


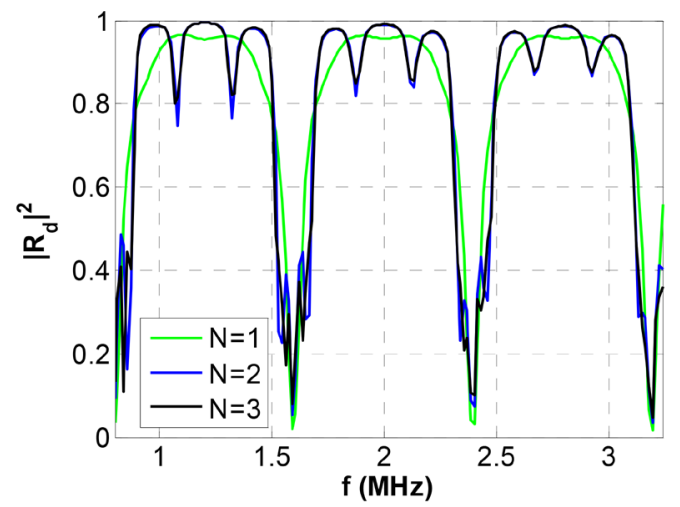

(a)

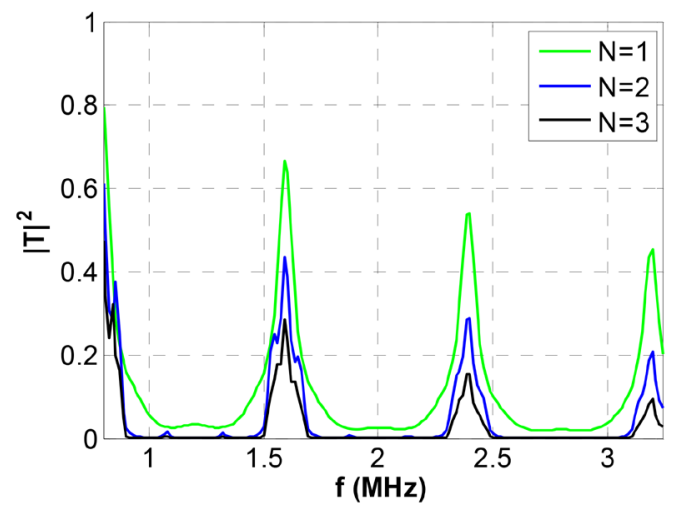

(c)

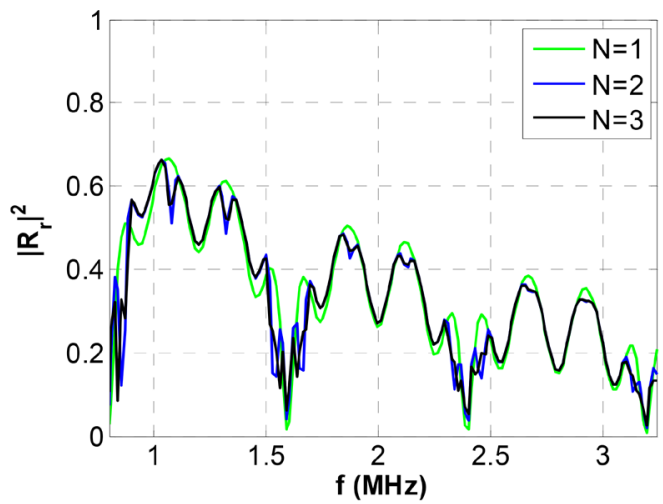

(b)

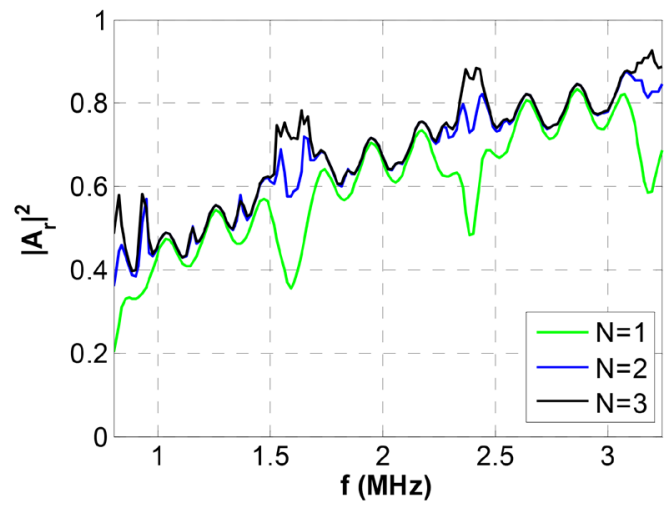

(d)

Figure 4. Reflection coefficients obtained with a (AL/PEg) stack, for $N=3$ periods: (a) direct reflection coefficient $\left|R_{d}\right|^{2}$, or (b) reverse reflection coefficient $\left|R_{r}\right|^{2}$, and associated (c) transmission coefficient $|T|^{2}$ and (d) reverse absorption coefficient $\left|A_{t}\right|^{2}$.

in Figure 4(a) and Figure 4(b) for the configurations (AL/PEg) and (PEg/AL), respectively. From $N=1$ period, the reflection minima linked to the propagating Lamb modes in the aluminum plate keep their positions for all configurations. These minima of reflection are observed at the frequencies 1.6, 2.4 and $3.2 \mathrm{MHz}$, in the bandwidth of the transducer.

The transmission coefficient illustrated by Figure 4(c), shows the forbidden band gaps as from $N=2$ periods, similarly as for the previously cited works [4] [6]. More precisely, the transmission coefficients (Figure 4(c)) exhibit a clear reduction of its amplitudes when the frequency and the number of periods increase. Thus, most of the wave energy is attenuated as the frequency and the number of periods increases. For example, if we take the case of the first peak at $1.6 \mathrm{MHz}$, the amplitude is equal to $0.63,0.41$ and 0.25 for $N=1,2$ and 3 periods, respectively. This leads us to deduce the absorption by the following relationship:

$$
\left|A_{\{d, r\}}\right|^{2}=1-|T|^{2}-\left|R_{\{d, r\}}\right|^{2}
$$

As a result, the absorption coefficients $\left|A_{\{d, r}\right|^{2}$ also depend on the insonation side. The estimated absorption on the reverse side is equal to $0.33,0.57$ and 0.73 at $1.6 \mathrm{MHz}$ (Figure $4(\mathrm{~d})$ ). 


\subsection{Volume Fraction Effect for $N=3$}

Siryabe et al., [6] observed an increase in the oscillations amplitude of the reflection coefficients when the volume fraction ratio and frequency increase (Figure $5(\mathrm{a})$ ). In the case of our configuration (PEg/AL), for a fixed period $N=3$ (Figure 5(b)), a decrease of the oscillations amplitude of reflection coefficients with the volume fraction and the frequency is observed since they are directly linked to the acoustic impedance contrast. This divergence can be explained by the fact that in the case of our configuration, the acoustic impedance contrast is lower than the case of study carried out by Siryabe et al., where air properties were neglected. In addition, we can observe that the minima are periodically spaced out by a shift of frequency $\Delta f=c_{L} /(2 d)$ such as $\Delta f_{P E C}=[267,242,221]$ $\mathrm{kHz}$ for $\mathrm{PEg} / \mathrm{AL}$ configuration, corresponding to the longitudinal velocities $c_{L, P E C}$ $=[2133,1933,1769] \mathrm{m} / \mathrm{s}$, respectively associated to the volume fraction $V_{f}=$ $20 \%, 40 \%$ and $60 \%$.

\subsection{Effect of Disbond Simulated by a Thin Teflon Plate}

The insertion of a thin Teflon layer of which properties are given in Table 3, within two adjacent plates ( $\mathrm{AL}$ and $\mathrm{PE}$ ), increases the layer number of the period from two to three. The presence of 100 to $500 \mu \mathrm{m}$ Teflon thickness is analyzed. We observed a shift to low frequencies on the local maxima and minima of reflection. The maximum shift is around $250-300 \mathrm{kHz}$. As illustrated by Figure 6, there are not significant changes depicted on the minima of reflection. By increasing the Teflon thickness, undulations on the transmission spectra increase too. Furthermore, reflection coefficients still have approximately same amplitude, whatever the number of period increases (Figure 6). About the location of the forbidden bands in the periodic structure, as we can see in Figure 6(c), three forbidden bands in the frequency range $[0.75 ; 3.25] \mathrm{MHz}$ are observed: from 1 to $1.5 \mathrm{MHz}, 1.7$ to $2.3 \mathrm{MHz}$ and 2.5 to $\mathrm{MHz}$. As an illustration, the influence of the variations of Teflon thickness layer on these passbands is depicted in Figure 6(c) where narrow passbands are observed in the frequency range from 1 to $1.5 \mathrm{MHz}$, reducing the forbidden bands.

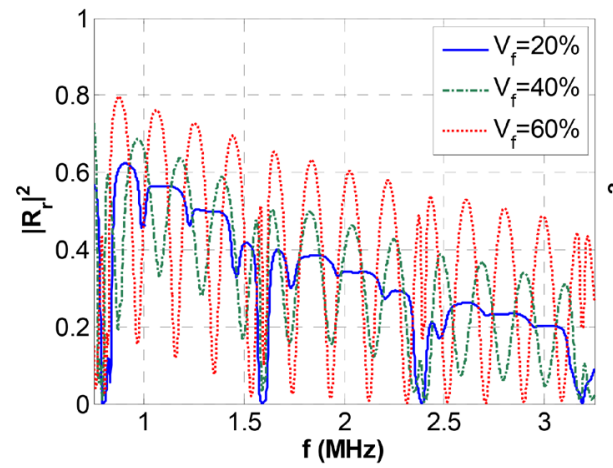

(a)

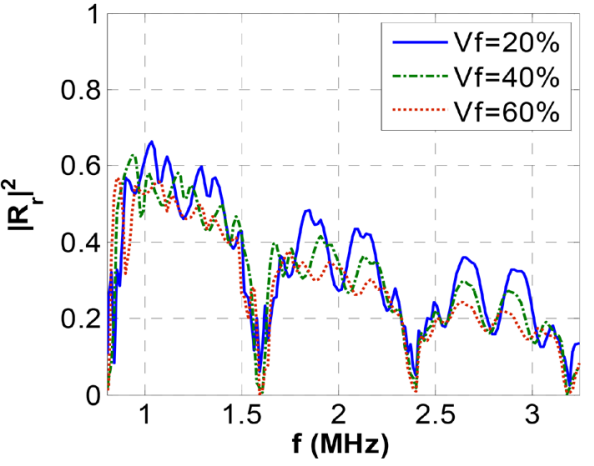

(b)

Figure 5. Effects of volume fraction on the reflection coefficients (a) (PEp/AL) configuration [6] and (b) (PEg/AL) configuration for $N=3 ; V_{f}=20 \%, 40 \%, 60 \%$. 


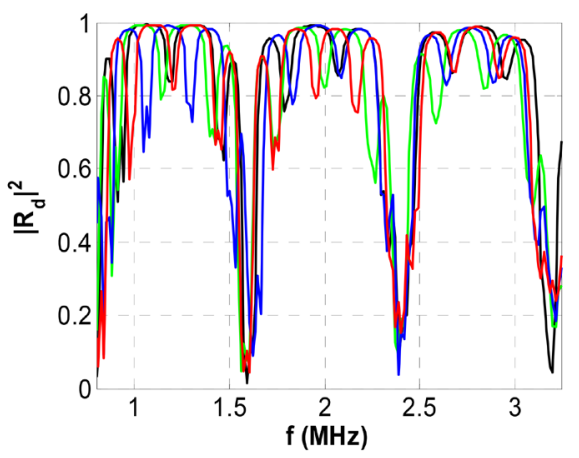

(a)

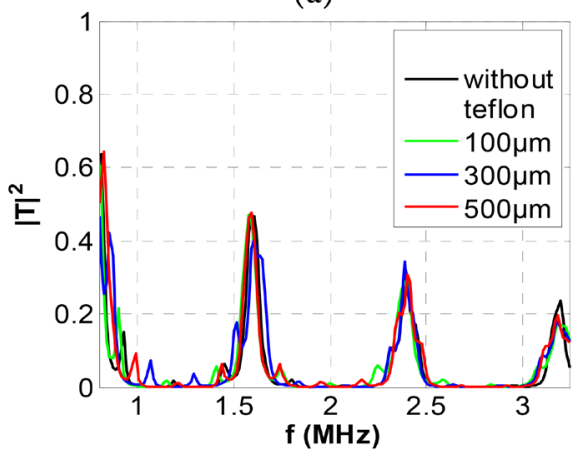

(c)

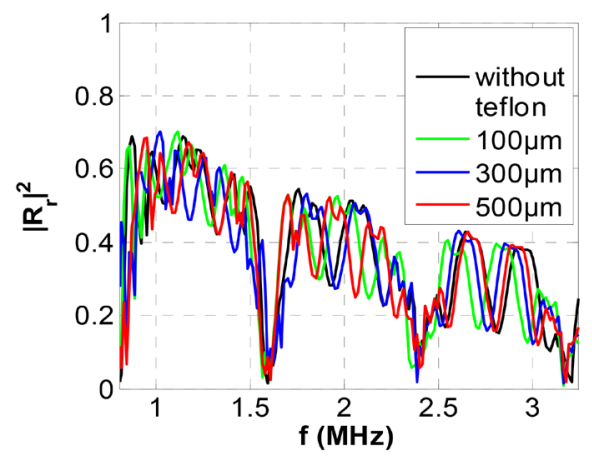

(b)

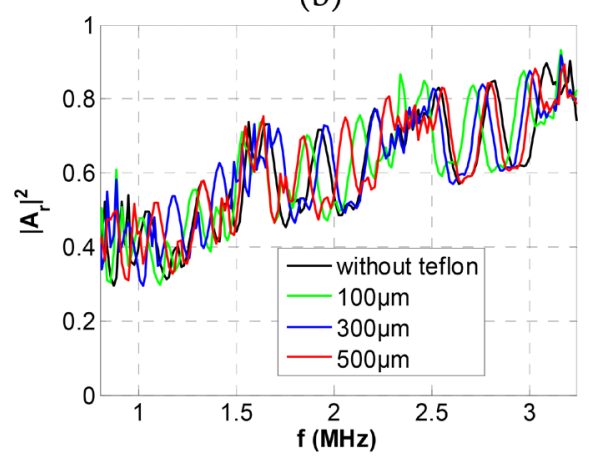

(d)

Figure 6. Effects of thin Teflon plate between $\mathrm{AL}$ and $\mathrm{PE}$ plate for $N=3$ (a) direct (AL/PE) and $(\mathrm{b})$ reverse $(\mathrm{PE} / \mathrm{AL})$ configuration [6].

\subsection{Number of Layer of Period Effect: Reference Case of $(\mathrm{AL} / \mathrm{PEg} / \mathrm{PEg} / \mathrm{AL}) \times N$ and Symmetric Case of $(\mathrm{PEg} / \mathrm{AL} / \mathrm{AL} / \mathrm{PEg}) \times N$}

In the structure of type $\mathrm{AL} / \mathrm{PEg}$, the reflection coefficients reach their maximum from the second period $(N=2)$. It is not the case of the structure of type $\mathrm{AL} / \mathrm{PEg} / \mathrm{PEg} / \mathrm{AL}$, where the reflection coefficients do not evolve anymore and this from the period $N=1$ (Figure 7 (a) and Figure 7(b)). This is due to the fact that, the number of layers forming one period has been increased, but also to their arrangements. On the one hand, this result means that the reflection coefficient is related only to the first layers of the multilayer structure, the following ones being dissimulated by the attenuation effect. On the other hand, in the reverse configuration $\mathrm{PEg} / \mathrm{AL} / \mathrm{AL} / \mathrm{PEg}$ (Figure $7(\mathrm{~b})$ ), the more the frequency increases, the more the amplitude of the reflection coefficients decrease. We also observe a duplication of the spectrum width with the apparition of the news minima of reflection, due to the double thickness of the aluminum layer.

Concerning the transmission coefficients, depending on the reference stack configuration $(\mathrm{AL} / \mathrm{PEg} / \mathrm{PEg} / \mathrm{AL}) \times N$ (Figure $7(\mathrm{a})$ ) or the symmetric one $(\mathrm{PEg} / \mathrm{AL} / \mathrm{AL} / \mathrm{PEg}) \times N($ Figure $7(\mathrm{~b}))$, the insonation side influences significantly the evolution of the transmission coefficients. The forbidden band gap appears at a period $N=1$ for the reference configuration (Figure $7(\mathrm{c})$ ), and from $N \geq 2$ periods in the symmetric case (Figure 7 (d)). This leads us to conclude that, in the reference configuration case $(\mathrm{AL} / \mathrm{PEg} / \mathrm{PEg} / \mathrm{AL})$, the more the layer number 


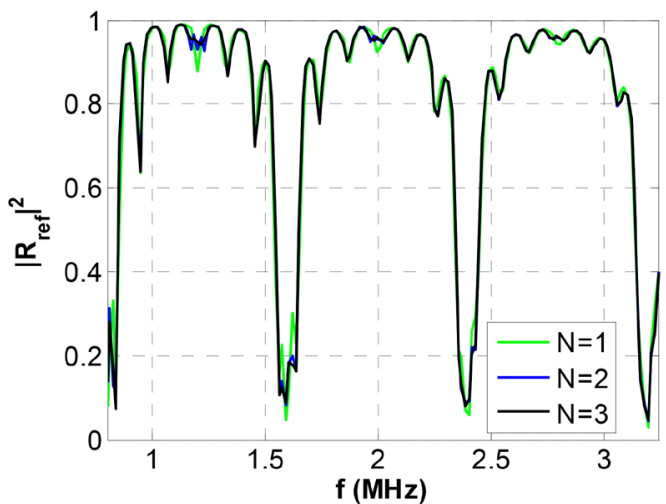

(a)

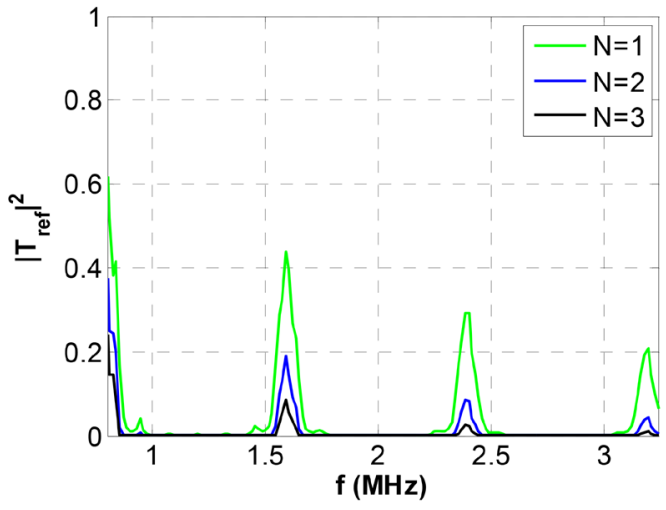

(c)

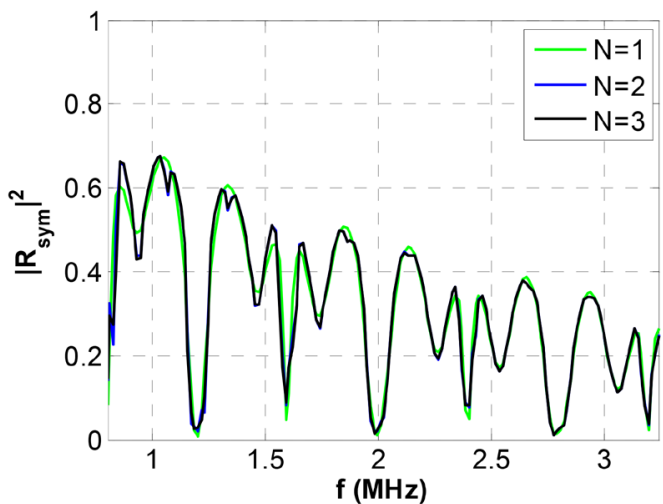

(b)

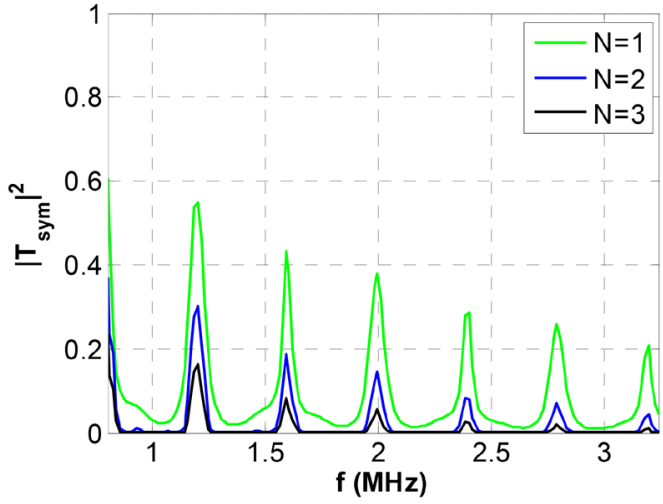

(d)

Figure 7. Coefficients obtained in (a), (b) reflection and (c), (d) transmission for the (a), (c) $\mathrm{AL} / \mathrm{PEg} / \mathrm{PEg} / \mathrm{AL}$ and (b), (d) PEg/AL/AL/PEg configurations for $N=1,2,3 ; V_{f}=20 \%$.

of a period increases, the more the forbidden bands are observed in the multilayer. Moreover, amplitudes of the maxima of transmission coefficients decrease considerably when the frequency and the number of period increase. Regarding the number of peaks, it shifted from $n_{\text {peaks }}$ to $2 n_{\text {peaks }}$ for the reference configuration versus the symmetric one, respectively. In the reference configuration, the position of the maximum of transmission is the same as for the case of $\mathrm{AL} / \mathrm{Peg}$, what is to be understood as the resonances of the first AL layer. In the symmetric configuration, additional maxima are observed at 1.2, 2.0 and $2.8 \mathrm{MHz}$, in between to the three maxima of transmission of the reference stack, respectively. Moreover, in the reference configuration, the positions and widths of the forbidden bands are identical to those of the AL/PEg configuration.

\section{Conclusion}

In this study, the effect of grease inclusion, Teflon, volume fraction of grease and the number of layers composing one period were highlighted by the analysis of the reflection and transmission spectra of an immersed multilayered media. As in the porous case of AL/PEp, reflection coefficients reach their maximum as from the second period $(N=2)$, in the case of AL/PEg, the forbidden bands gap appears in the multilayer stack as from $N=2$ periods. In addition, in the case of 
the studied (PEg/AL) configuration, for a fixed number of periods $N=3$, a decrease of the oscillations amplitude of the reflection coefficients with the volume fraction and the frequency is observed, since they are directly linked to the acoustic impedance contrast. The presence of Teflon defects of 100 to $500 \mu \mathrm{m}$ thick was analyzed. As a result, a shift is observed to low frequencies on the local maxima and minima of reflection. The maximum shift was around $250-300 \mathrm{kHz}$. Finally, concerning the transmission coefficients, unlike to the structures of type (AL/PE or PE/AL) [4], (AL/PEp or PEp/AL) [6] and (AL/PEg or PEg/AL) where the transmission spectra are identical whatever the insonation side (direct or reverse). In the case where the elementary stack consists of four layers, the forbidden band gaps appear at a period $N=1$ for the reference configuration and from $N \geq 2$ periods in the symmetric case. The number of peaks, shifted from $n_{\text {peaks }}$ to $2 n_{\text {peaks }}$ for the reference and symmetric configurations, respectively. Further studies are to be led experimentally in order to validate those numerical results. It would also be interesting to include piezoelectric layers as dynamic modulators of the reflection and transmission coefficients. As a perspective, such piezo-active and controllable multilayers have many potential applications as actuators and sensors in the industry. We could plan to evaluate the electromechanical response of the obtained global structure.

\section{Conflicts of Interest}

The authors declare no conflicts of interest regarding the publication of this paper.

\section{References}

[1] Khelif, A., Choujaa, A., Djafari-Rouhani, B., Wilm, M., Ballandras, S. and Laude, V. (2003) Trapping and Guiding of Acoustic Waves by Defect Modes in a Full-BandGap Ultrasonic Crystal. Physical Review B, 68, Article No. 214301. https://doi.org/10.1103/PhysRevB.68.214301

[2] Perez, S., Caballero, J.V., Martinez-Sala, R., Rubio, C., Sanchezdehesa, J., Meseguer, F., Llinares, J. and Galvez, F. (1998) Sound Attenuation by a Two-Dimensional Array of Rigid Cylinders. Physical Review Letters, 80, Article No. 5325. https://doi.org/10.1103/PhysRevLett.80.5325

[3] Rokhlin, S.I. and Wang, L. (2002) Stable Recursive Algorithm for Elastic Wave Propagation in Layered Anisotropic Media: Stiffness matrix Method. The Journal of the Acoustical Society of America, 112, 822-834. https://doi.org/10.1121/1.1497365

[4] Lenoir, O. and Maréchal, P. (2009) Study of Plane Periodic Multilayered Viscoelastic Media: Experiment and Simulation. IEEE International Ultrasonics Symposium Proceedings, Rome, 20-23 September 2009, 1028-1031. https://doi.org/10.1109/ULTSYM.2009.5441518

[5] Lenoir, O., Maréchal, P. and Rembert, P. (2009) Study of Period and Structure Modes of Periodic Multilayers with the S Matrix Formalism. 19ème Congrès Français de Mécanique, Marseille, 24-28 August 2009, 6 p.

[6] Siryabe, E., Ntamack, G. and Maréchal, P. (2013) Holes Effects in Plane Periodic Multilayered Viscoelastic Media. Open Journal of Acoustics, 3, 80-87. 
https://doi.org/10.4236/oja.2013.33013

[7] Maréchal, P., Lenoir, O., Khaled, A., Ech Cherif El Kettani, M. and Chenouni, D. (2014) Viscoelasticity Effect on a Periodic Plane Medium Immersed in Water. Acta Acustica United with Acustica, 100, 1036-1043. https://doi.org/10.3813/AAA.918783

[8] Maréchal, P. and Lenoir, O. (2010) Effets de dépériodisation dans une structure multicouche plane viscoélastique: expérience et simulation. 10ème Congrès Français d'Acoustique, Lyon, 12-16 April, 2010, 6 p. https://hal.archives-ouvertes.fr/hal-00541707/document

[9] Lenoir, O., Khaled, A., Marechal, P. and Chenouni, D. (2012) Effects of a Default on the Reflectivity of Plane Periodic Media. Proceedings of the Acoustics, Nantes, 23-27 April 2012, 271-276.

[10] Khaled, A., Elamri, F.-Z., El Kadmiri, I. and Bria, D. (2019) Effects of Defect Layers Insertion on the Transmission of a Submerged One-Dimensional Phononic Structure. International Conference on Wireless Technologies, Embedded and Intelligent Systems, Fez, Morocco, 3-4 April 2019, 1-6. https://doi.org/10.1109/WITS.2019.8723782

[11] Magoariec, H. (2003) Adaptation élastoplastique et homogénéisation périodique. Ph.D. Thesis, Aix-Marseille II University, Marseille.

[12] Caloz, C. and Itoh, T. (2006) Electromagnetic Metamaterials: Transmission Line Theory and Microwave Applications: The Engineering Approach. Wiley \& IEEE Press, Hoboken, NJ. https://doi.org/10.1002/0471754323

[13] Gaunaurd, G., Callen, E. and Barlow, J. (1984) Pressure Effects on the Dynamic Effective Properties of Resonating Perforated Elastomers. The Journal of the Acoustical Society of America, 76, 173-177. https://doi.org/10.1121/1.391090

[14] Hashin, Z. and Shtrikman, S. (1963) A Variational Approach to the Theory of the Elastic Behaviour of Multiphase Materials. Journal of the Mechanics and Physics of Solids, 11, 127-140. https://doi.org/10.1016/0022-5096(63)90060-7

[15] Hashin, Z. (1965) On Elastic Behaviour of Fibre Reinforced Materials of Arbitrary Transverse Phase Geometry. Journal of the Mechanics and Physics of Solids, 13, 119134. https://doi.org/10.1016/0022-5096(65)90015-3

[16] Wang, L. and Rokhlin, S.I. (2001) Stable Reformulation of Transfer Matrix Method for Wave Propagation in Layered Anisotropic Media. Ultrasonics, 39, 413-424. https://doi.org/10.1016/S0041-624X(01)00082-8 